\title{
Periodic variations of GCR intensity and anisotropy related to solar rotation by ACE/CRIS, STEREO, SOHO/EPHIN and neutron monitors observations
}

\author{
R. Modzelewska $a^{*}$ and A. Gila,b \\ a Faculty of Exact and Natural Sciences, Institute of Mathematics, Siedlce University, \\ Konarski Str. 2 08-110 Siedlce, Poland \\ b Space Research Centre, Polish Academy of Sciences, Bartycka Str.18A, 00-716 Warsaw, Poland \\ E-mail: renatam@uph.edu.pl, gila@uph.edu.pl
}

\begin{abstract}
:
We study the periodic variations of galactic cosmic rays (GCRs) related to solar rotation based on neutron monitor (NM), ACE/CRIS, STEREO and SOHO/EPHIN measurements, in solar minima 23/24 and 24/25 characterized by the opposite polarities of solar magnetic cycle. Now there is an opportunity to re-analyze the polarity dependence of the amplitudes of the recurrent GCR variations in 2007-2009 for negative A $<0$ solar magnetic polarity and to compare it with the clear periodic variations related to solar rotation in 2017 2019 for positive A $>0$. We use the Fourier analysis method to study the periodicity in the GCR fluxes. Since the GCR recurrence is a consequence of solar rotation, we analyze not only GCR fluxes, but also solar and heliospheric parameters examining the relationships between the 27-day GCR variations and heliospheric, as well as, solar wind parameters. We find that the polarity dependence of the amplitudes of the 27-day variations of the GCR intensity and anisotropy for NMs data is kept for the last two solar minima: 23/24 (2007-2009) and 24/25 (2017-2019) with greater amplitudes in positive A $>0$ solar magnetic polarity. ACE/CRIS, SOHO/EPHIN and STEREO measurements are not governed by this principle of greater amplitudes in positive A $>0$ polarity. GCR recurrence caused by the solar rotation for low energy $(<1 \mathrm{GeV})$ cosmic rays is more sensitive to the enhanced diffusion effects, resulting in the same level of the 27-day amplitudes for positive and negative polarities. While high energy $(>1 \mathrm{GeV})$ cosmic rays registered by NMs, are more sensitive to the large-scale drift effect leading to the 22-year Hale cycle in the 27-day GCR variation, with the larger amplitudes in the $\mathrm{A}>0$ polarity than in the $\mathrm{A}<0$.
\end{abstract}

37th International Cosmic Ray Conference (ICRC 2021)

July 12th-23rd, 2021

Online-Berlin, Germany

\section{*Presenter}

(c) Copyright owned by the author(s) under the terms of the Creative Commons 


\section{Introduction}

The 27-day variation of the GCR intensity and anisotropy, related to the solar rotation, is connected with the heliolongitudinal asymmetry of the heliospheric parameters being under the influence of corotating interaction regions (CIRs), for review see $[1,2]$

In this paper we extend the quantitative study of the 27-day variation of the GCR intensity for lower part of the energy spectrum using spacecraft data: SOHO/EPHIN, STEREO and ACE/CRIS and compare it with NMs observations, see details in [3]. It is now an opportunity to re-analyze the polarity dependence of the amplitudes of the recurrent GCR variations in solar minimum 23/24 in 2007-2009 when $\mathrm{A}<0$ and to compare it with the clear periodic variations related to solar rotation in solar minimum 24/25 in 2017-2019 when $\mathrm{A}>0$.

\section{Experimental Data and Methods}

We analyze the daily data of GCR proton flux for SOHO/EPHIN, STEREO A and B for solar minimum 23/24 when $\mathrm{A}<0$ in 2007-2009 and solar minimum 24/25 when $\mathrm{A}>0$ in 2017-2019. STEREO B data are not available in 2017-2019. The same we perform for ACE/CRIS GCR fluxes of Carbon, Nitrogen, Oxygen, Neon, Silicon and Iron. Additionally we update the calculations for the daily GCR intensity and anisotropy for NMs with different cut off rigidities, from $0.67 \mathrm{GV}$ to 4.00 GV: Hermanus, Newark, Kerguelen, Oulu and Apatity. We study the dynamics of the recognized periodicity connected with the solar rotation using Lomb periodogram [4] adapting the procedure from [5]. We calculate the power of the periodic variation connected with the Sun's rotation for period of $27+/-2$ days with $95 \%$ confidence level. Maximal power from Lomb periodogram indicates the main period of the recurrent GCR variations in the analyzed time series. As an example Figure $1\left(1^{\text {st }}\right.$ panel) presents STEREO A daily proton fluxes for kinetic energy $40-$ $60 \mathrm{MeV}$ and 60-100 MeV for solar minimum 23/24 when $\mathrm{A}<0$ in 2007-2009. The second panel of Figure 1 presents normalized and detrended (by excluding 29 days running average) GCR intensity. The timeline of the power of recurrent variations lasting 25-29 days and the recognized main period for each Bartel rotation (BR) are displayed on the third and fourth panels of Figure 1, respectively. Figure 2 presents data and Lomb analysis results of STEREO A for solar minimum $24 / 25$ when $A>0$ in 2017-2019.

Figures 1 and 2 demonstrate the existence of the periodic variations of the GCR intensity related to solar rotation for the last two solar minima with opposite solar magnetic polarity: $23 / 24$ in 2007-2009 and 24/25 in 2017-2019. One can observe the high power of the very famous episode of the 27-day variation when $\mathrm{A}<0$ in 2007-2008 (Fig. 1) and clear periodic variations when A $>$ 0 in 2017-2018 (Fig. 2) for both energy bins. The same data processing was done for STEREO B, $\mathrm{SOHO} / \mathrm{EPHIN}, \mathrm{ACE} / \mathrm{CRIS}$ and NMs data revealing similar results. 

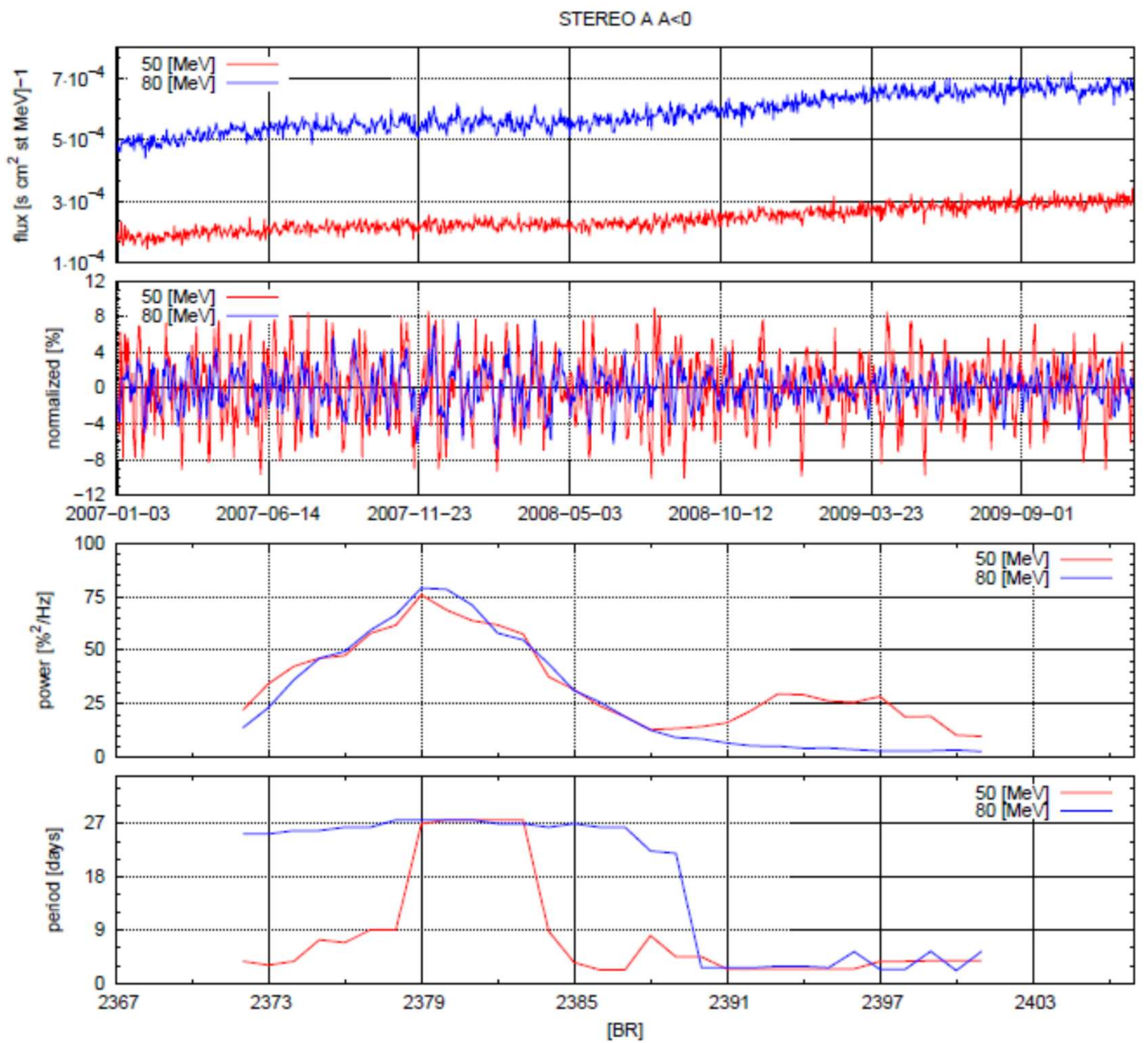

Fig. 1. (1st panel) STEREO A daily proton fluxes for kinetic energy 40-60 MeV and $60-100 \mathrm{MeV}$ for solar minimum 23/24 when $A<0$ in 2007-2009; ( 2 nd panel) GCR intensity normalized and detrended by excluding 29 days running average; the timeline of the power of recurrent variations lasting 25-29 days (3rd panel) and the recognized main period (4th panel). 

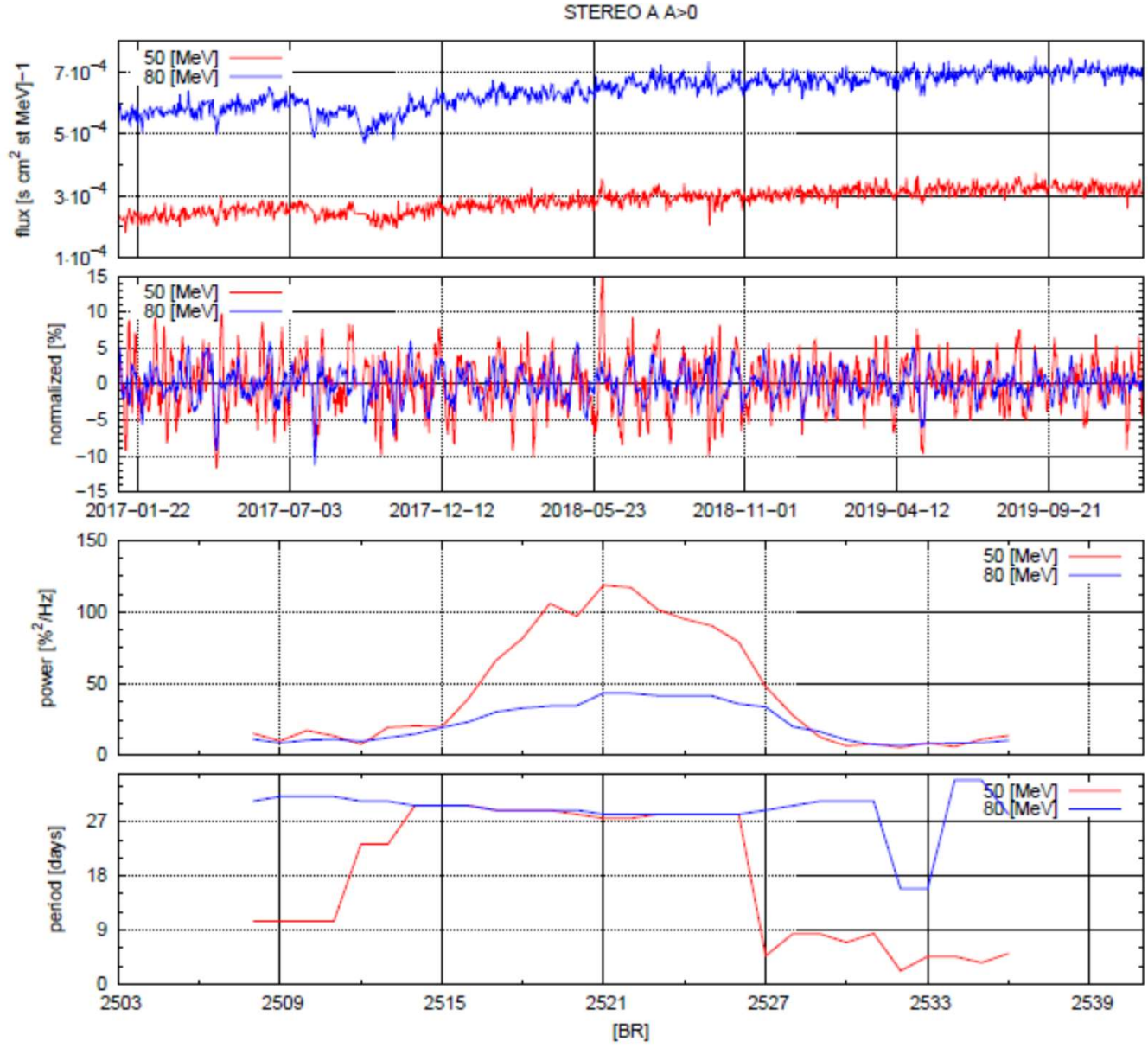

Fig. 2. The same as in Fig. 1 for solar minimum 24/25 when $A>0$ in 2017-2019
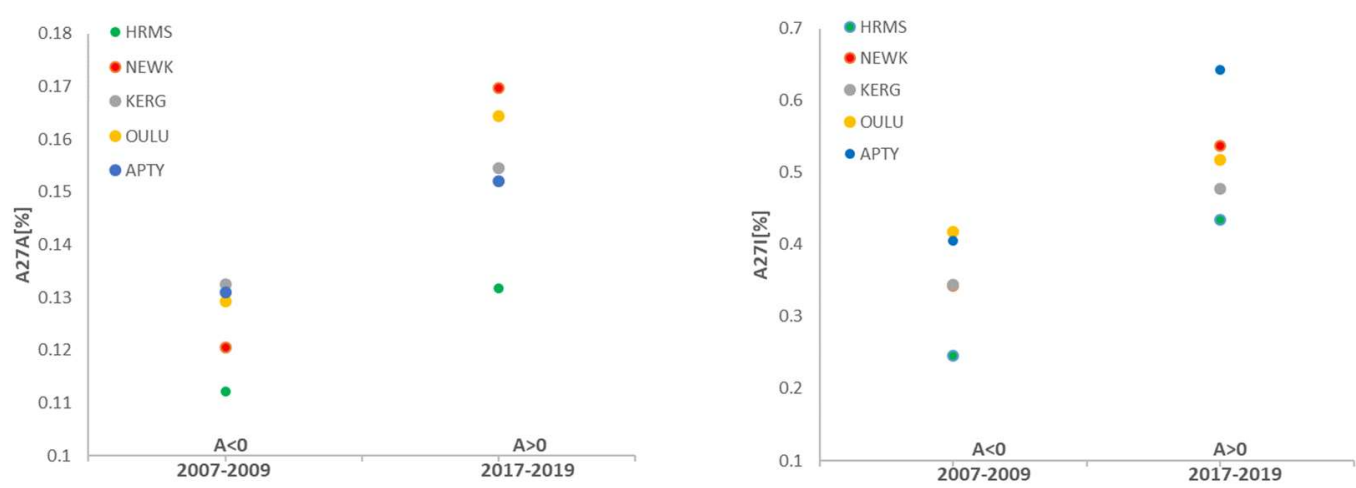

Fig. 3. Amplitude of the 27-day variation of the GCR anisotropy (left) and intensity (right) observed by NMs (Hermanus, Rc = 4:58GV; Newark, Rc = 2:40GV, Kerguelen, Rc = 1:14GV; Oulu, Rc = 0:81GV; Apatity, Rc = 0:65 GV) for the solar minimum 23/24 when $\mathrm{A}<0$ in 2007-2009 and for the solar minimum 24/25 when A $>0$ in 2017-2019. 


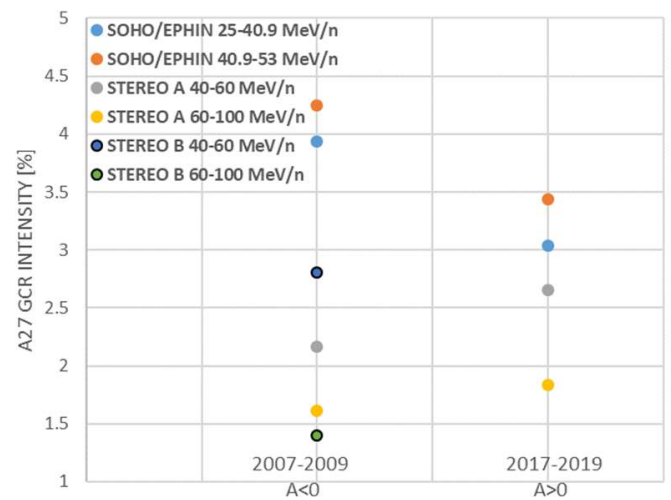

Fig. 4. Same as in Fig. 3 for the 27-day variation of the GCR proton intensity recorded by SOHO/EPHIN and STEREO $\mathrm{A}$ and $\mathrm{B}$
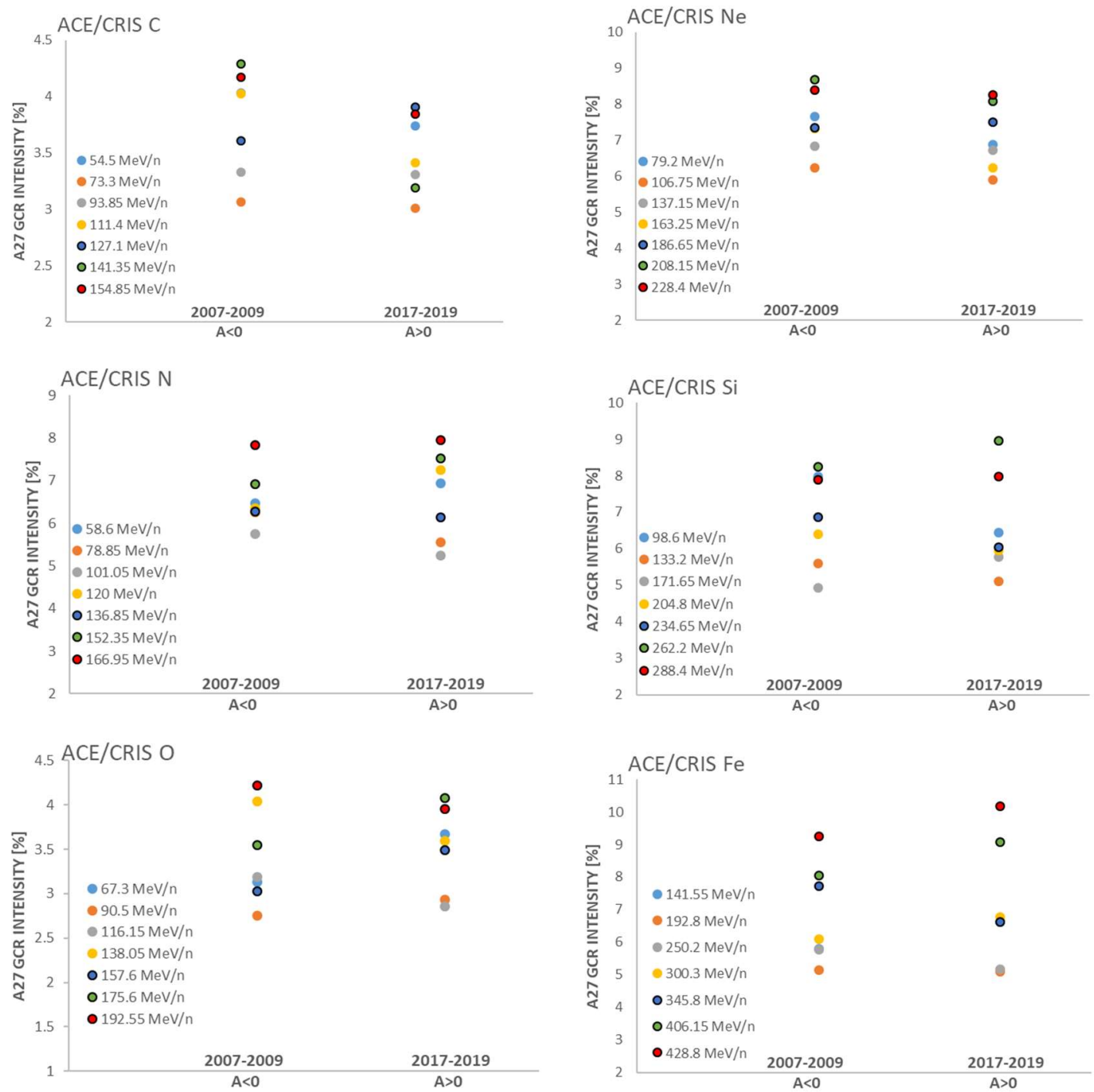

Fig. 5. Same as in Fig. 4 for GCR carbon, nitrogen, oxygen, neon, silicon and iron recorded by ACE/CRIS 


\section{Data analysis}

First we study the polarity dependence of the amplitude of the 27-day variation using NMs data: Hermanus, Newark, Kerguelen, Oulu and Apatity. We update the calculations for the 27-day variation of the GCR anisotropy A27A [5] and intensity A27I [6] for the solar minima 23/24 and 24/25. The average amplitudes of the A27A and A27I for the last two solar minima are presented in Figure 3. We find that the average A27A and A27I are polarity dependent, with greater amplitudes when $\mathrm{A}>0$ in 2017-2019 for solar minimum 24/25 than for $\mathrm{A}<0$ in 2007-2009 for solar minimum 23/24. Figure 4 presents the average A27 of the GCR proton intensity for SOHO/EPHIN (energy bins: 25-40.9 MeV/n and 40.9-53 MeV/n) and STEREO A, B (energy bins: $40-60 \mathrm{MeV} / \mathrm{n}$ and $60-100 \mathrm{MeV} / \mathrm{n}$ ) for $\mathrm{A}>0$ and $\mathrm{A}<0$ polarity. Although, the picture in Figure 4 is quite complicated, the weak tendency of greater amplitudes for $\mathrm{A}>0$ than for $\mathrm{A}<0$ for higher energies $40-100 \mathrm{MeV} / \mathrm{n}$ in STEREO data can be seen, but opposite scenario is noticeable for lower energies $25-53 \mathrm{MeV} / \mathrm{n}$ in SOPHO/EPHIN data. Figure 5 presents the A27 of GCR fluxes for Carbon, Nitrogen, Oxygen, Neon, Silicon and Iron by ACE/CRIS, respectively. For each species we analyse 7 energy bins available at ACE/CRIS measurements. Values of the A27 for GCR flux of heavier nuclei registered by ACE/CRIS remain on the same level for both polarities for all considered species. For more details please see [3].

\section{Summary}

We confirm the polarity rule in the behaviour of the amplitudes of the 27-day variations of the GCR anisotropy and intensity observed by NMs in the solar minima: 23/24 (2007-2009) and 24/25 (2017-2019), namely larger amplitudes are observed for A $>0$ polarity epoch. The amplitudes of the 27-day variations of the GCR intensity observed by ACE/CRIS, STEREO A, B and SOHO/EPHIN, in the solar minima 23/24 and 24/25 remain at the same level and do not seem to be polarity dependent. Recurrent variations connected with the solar rotation for low energy $(<1 \mathrm{GeV})$ cosmic rays are more sensitive to the enhanced diffusion effects, leading to the same level of the 27-day amplitudes for $\mathrm{A}>0$ and $\mathrm{A}<0$ polarities. Whereas high energy $(>1 \mathrm{GeV})$ cosmic rays observed by NMs, are more sensitive to the large-scale drift effect resulting in the 22-year Hale cycle of the 27-day GCR variations, with the larger amplitudes in the A $>0$ polarity than in the $\mathrm{A}<0$. Nevertheless, processes around CIR are more complex and need further study, e.g. because of competition between modulation and acceleration of cosmic rays around stream interaction regions.

\section{References}

[1] Richardson, I.G. 2004, SSR, 111, 267

[2] Richardson, I.G. 2018, Living Rev. Solar Phys., 15, 1

[3] R. Modzelewska, R. A. Gil, A., 2021, Astronomy \& Astrophysics, 646, A128

[4] Lomb, N.R. 1976, Astrophys. Space Sci., 39, 447

[5] Modzelewska, R., Alania, M.V. 2013, Sol. Phys., 286, 593

[6] Gil, A., Mursula, K. 2017, Astronomy \& Astrophysics, 599, A112 